\title{
Multiobjective Optimized Bipartite Matching for Resource Allocation
}

\author{
Fanglei Sun, Victor O. K. Li \\ Department of Electrical and Electronic Engineering \\ University of Hong Kong \\ Pokfulam Road, Hong Kong, China \\ Email:\{flsun, vli\}@eee.hku.hk
}

\author{
Zhifeng Diao \\ Department of Electrical Engineering \\ Arizona State University \\ AZ 85278 USA \\ Email:zhifeng.diao@asu.edu
}

\begin{abstract}
The Hungarian algorithm can provide the maximum weighted bipartite matching for assignment problems. However it can only solve the single objective weight optimization problem. In this paper, a modified bipartite matching (MBM) algorithm is proposed to solve the weighted bipartite matching problem with multiobjective optimization. In addition, our MBM algorithm is applicable to asymmetric bipartite graph, which is common in resource allocation problems. We illustrate the application of MBM to antenna assignments in wireless multipleinput multiple-output (MIMO) systems for both symmetric and asymmetric scenarios. The simulation results show that MBM enjoys low computational complexity and maximizes the system capacity, while keeping the fairness among mobile users.
\end{abstract}

\section{INTRODUCTION}

The Hungarian method for the assignment problem describe an algorithm for constructing a maximum weight perfect matching in a bipartite graph [1]. It is proved that the Hungarian algorithm can always find the maximum assignment, i.e., an optimal solution to the maximum weight sum [1]. Compared with exhaustive search, the computational complexity is reduced to $O\left(n^{4}\right)$, where $\mathrm{n}$ is the cardinality (the number of the elements in a set) of the node set of a bipartite graph. However, the Hungarian method only considers single objective optimization, limiting its application to assignments with multiobjective optimization. In addition, for asymmetric bipartite graphs, the Hungarian algorithm only considers the assignments based on the minimum cardinality of the bipartite node sets. It will cause unnecessary resource wastage when the redundant resource is reusable.

In network design, a single global objective, which may be one of the network performance measures, such as throughput, is often examined to optimize network performance. This approach is meaningful in the homogeneous situation, such as when each user has the same quality of service (QoS) requirement. In this case, equal performance is the implied fairness criterion, and global optimality is equivalent to individual optimization. However, in the heterogeneous case, individual requirements may be ignored by using a single overall objective, and sometimes users may have to sacrifice

This research is supported in part by the Research Grants Council of the Hong Kong Special Administrative Region, China, under Grant No. HKU 7148/06E. their own performance for the good of the entire network [2] [3].

In wireless multiple-input multiple-output (MIMO) communication systems based on spatial multiplexing, a data stream is split into multiple parallel substreams, and each substream is transmitted through one of the transmit antennas [4]. Combined with multiuser diversity [5] in cellular packet transmission systems, a couple of antenna assignment schemes have been proposed for packet schedulers to maximize the system capacity or to provide fairness among mobile users [6][8]. However these scheduling schemes only consider single objective optimization. None of them considers multiobjective optimization which account for both capacity maximization and fairness.

In this paper, a modified bipartite matching (MBM) algorithm is proposed for multiobjective optimization, such as maximizing the total throughput, while keeping the fairness among individual users. In MBM, this is realized by applying the Hungarian algorithm to each updated bipartite graph and choosing the matching with the largest fairness index among the updated matchings. The bipartite graph is updated by reducing one edge at a time till no edges remain in the graph. The deleted edge is the one violating the fairness criterion most seriously. Consequently, the fairness comparison can be finished in $O\left(n^{2}\right)$. For asymmetric bipartite graphs, i.e. the number of nodes in the two bipartite sets are different, if the resource to be assigned may be fully utilized, our MBM is designed to maximize the resource usage by reconstructing the graph and finding updated matchings. In this paper, MBM is applied to a MIMO communication system as an example. Simulation results show that it can effectively guarantee the system capacity and fairness performance whether or not the number of mobile users and the number of antennas are the same. More importantly, our proposed MBM algorithm can be widely applied to job assignments and other resource allocation problems which need multiobjective optimization.

The paper is organized as follows. Section II presents the details of the MBM algorithm. In Section III, we give an example of MBM for antenna assignments in wireless MIMO communication systems. Section VI draws the conclusions of this paper. 


\section{DESCRIPTION OF MBM}

\section{A. Multiobjective Optimization Functions}

A graph is denoted by $G(V, E)$, where $V$ is the vertex set and $E$ is the edge set of the graph. If $V=V_{1} \cup V_{2}$ with $V_{1} \cap V_{2}=\Phi$, and each edge in $E$ has one endpoint in $V_{1}$ and the other in $V_{2}$, the graph $G(V, E)$ is a bipartite graph, which can also be denoted as $G\left(V_{1}, V_{2}, E\right)$. The bipartite graph is very useful for some applications, such as an assignment problem which can be depicted as follows: Given a weighted complete bipartite graph $G=(X \cup Y, X \times Y)$, where edge $(x, y)$ has weight $w(x, y)$, find a matching $M$ from $X$ to $Y$ with maximum weight. In an application, $X$ could be a set of workers, $y$ a set of jobs, and $w(x, y)$ the profit made by assigning worker $x$ to job $y$. The essence of the assignment problem is to find the optimal matching. Basically, there are two approaches to the optimization problem: single objective overall weight optimization and multiobjective individual optimization.

For the overall weight optimization, we have a single objective optimization problem:

$$
\max \sum_{x \subset X, y \subset Y} w(x, y), \quad(x, y) \subset E .
$$

This approach is often used to obtain the optimal performance for the entire assignment, and the meaning of optimization is straightforward. To consider the individual performance, we resort to the multiobjective approach. For example, the system is required to keep profit fairness among the workers. Consider any matching $M_{j}(j=1, \ldots n)$, where $n$ is the maximum number of matchings chosen from $G$. We assume the objective evaluation function is $f\left(w_{j}(x, y)\right)$, where $(x, y) \subset M_{j}$ and $w_{j}(x, y)$ represents the weights of edges in $M_{j}$. Consequently, the multiobjective optimization problem is to combine (1) with

$$
\begin{array}{cc}
\max f\left(w_{j}(x, y)\right), \quad(x, y) \subset M_{j}, \\
j=1, \ldots, n .
\end{array}
$$

The multiobjective functions can be constructed based on specific applications, and are not limited to examples we have given in this paper. The main contribution of our work is to introduce a modified bipartite matching algorithm to provide a solution to general multiojective optimization problems.

\section{B. MBM Algorithm}

Since individuals' objectives may conflict with each other, and may conflict with the overall optimization objective, a new modified bipartite matching algorithm is proposed. In this subsection, only the symmetric bipartite matching is discussed, and the asymmetric case is presented in the next subsection. It has been proved that the Hungarian algorithm can always find the maximum weight matching for a bipartite graph. That means it can provide an optimal solution to the above problem (1). The following is based on the description of the procedure of the Hungarian algorithm in [7] and [9]. Matrix $W=\left[w_{i j}\right]$

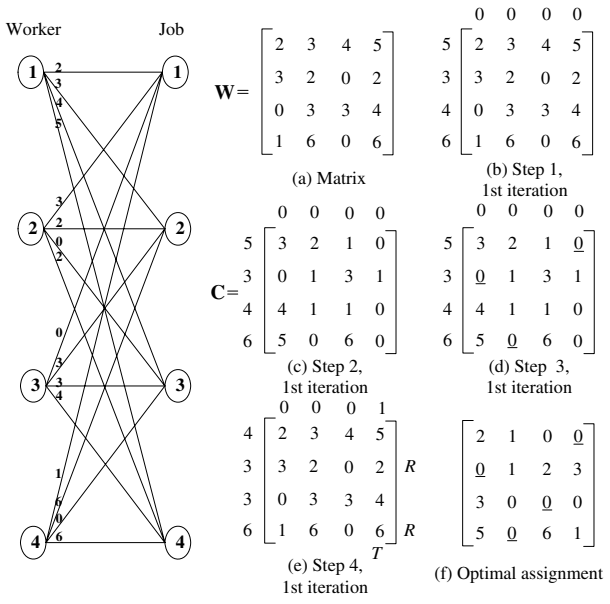

Fig. 1. Weighted bipartite matching.

has elements $w_{i j}$, which is the weight of assigning worker $i$ to job $j$ as shown in Fig. 1 (a).

1) Step 1: Let $X, Y$ be the bipartite sets. Initialize two labels $u_{i}$ and $v_{j}$ by $u_{i}=\max _{j} w_{i j}, v_{j}=0, i, j=1, \ldots, k$. In Fig. 1 (b), the numbers written at the left and the top of the matrix express the values of $u_{i}$ and $v_{j}$, respectively.

2) Step 2: Obtain the excess matrix $\mathbf{C}$ by the following: $c_{i j}=u_{i}+v_{j}-w_{i j}$. This is shown in Fig. 1 (c).

3) Step 3: Find the subgraph $G^{\prime}$ that includes vertices $i$ and $j$ satisfying $c_{i j}=0$ and the corresponding edge $e_{i j}$. Then find the maximum matching $M$ in $G^{\prime}$ and underline the entries in $M^{1}$. In this example, the maximum matching is found to be $(1,4),(2,1)$ and $(4,2)$. If $M$ is a perfect matching with $k$ edges, the optimal assignment is obtained. This is shown in Fig. 1 (d).

4) Step 4: Let $Q$ be a vertex cover of $G^{\prime}$, and let $R=X \cap Q$ and $T=Y \cap Q$. The vertex cover $Q$ is a vertex set of $G^{\prime}$ which contains at least one endpoint of each edge. In this example, $Q$ is chosen to be the nodes corresponding to Wroker 1 and 3, and Job 4. So $R$ corresponds to Worker 1 and 3 , and $T$ corresponds to Job 4. Now find $\epsilon=\min \left\{c_{i j}: x_{i} \in X-R, y_{j} \in Y-T\right\}$. For example, if $\epsilon$ equals 1 in Fig. 1, decrease $u_{i}$ by $\epsilon$ for the rows of $X-R$ and increase $v_{j}$ by $\epsilon$ for the columns of $T$. Then go to step 2 .

Steps 2 to 4 are repeated until the perfect matching $M$, i.e., the optimal assignment, is obtained.

After the procedure of the Hungarian algorithm, the maximum weighted matching $M$ can be achieved, and for simplicity, we denote the weights of the $k$ edges as $w_{i}, i=1, \ldots, k$. To consider the multiobjective fairness optimization problem (2), MBM calculates $\eta_{i}$ of the different weighted edges by

${ }^{1}$ There are various ways to find the maximum matching. See, for example [9]. 


$$
\eta_{i}=\left|w_{i}-\left(\sum_{i=1}^{k} w_{i}\right) / k\right|^{2}, \quad i=1, \ldots, k .
$$

In terms of the fairness, these $\eta_{i}$ values represent the distance from the mean. Then we choose the edges $m$ by

$$
\{m\}=\operatorname{argmax}_{i}\left\{\eta_{i}\right\}, \quad i=1, \ldots, k .
$$

For the original maximum matching, denoted as $M_{1}$, we can deduce that the edge $m$ violates the fairness criterion most seriously. So in order to improve the fairness performance, MBM deletes the edge $m$ in $G$, i.e. changing the corresponding element to zero in the matrix $W$. With the updated weight matrix, we apply the Hungarian algorithm again to find the new maximum matching. This is repeated until there is no edge left in $G$. For a square matrix $W$ with $n \times n$ elements, there are at most $O\left(n^{2}\right)$ iterations. Note that since there is no back-tracking in the algorithm to allow us to add back previously deleted edges, our solution does not guarantee the maximum matching. However, in our test cases, we find that the maximum matching is obtained most of the time. Given each obtained maximum matching $M_{j}(j=1, \ldots, n \times n)$, the corresponding fairness can be evaluated by the defined evaluation function $f\left(w_{j}(x, y)\right)$. Then the final solution given by $\mathrm{MBM}$ is

$$
\begin{aligned}
&\{p\}=\operatorname{argmax}_{j}\left\{f\left(w_{j}(x, y)\right)\right\}, \\
&(x, y) \subset M_{j}, j=1, \ldots, n \times n .
\end{aligned}
$$

For multiobjective optimization, based on the Hungarian algorithm, $M_{j}$ is guaranteed to be the maximum weighted matching for each updated bipartite graph. By deleting the edge with the most serious fairness violation in the previous matching, the fairness function is updated. By comparing the fairness performance in $O\left(n^{2}\right)$ calculations, the final solution $M_{p}$ can achieve the best fairness among those maximum weighed matchings for each updated $G$. Therefore, MBM can effectively solve the multiobjective optimization problem. In addition, if the system is given a fairness performance threshold $\xi$, the iterations will stop once the fairness performance of the updated matching is above $\xi$, so $O\left(n^{2}\right)$ is the upper bound of the computational complexity.

\section{Asymmetric Bipartite matching}

For a bipartite graph $G\left(V_{1}, V_{2}, E\right)$, if the cardinalities of $V_{1}$ and $V_{2}$, denoted as $n_{1}$ and $n_{2}$, are not equal, then this bipartite graph is asymmetric. Firstly, let consider the case when $n_{1}<$ $n_{2}$, that is, the resource to be assigned is not adequate for all users. Then the bipartite matching can be formulated as in Fig. 2. In this case, our MBM appends $\left(n_{2}-n_{1}\right)$ all-zero rows to the original weight matrix $W_{n_{1} \times n_{2}}$ to form a square weight matrix $W_{n_{2} \times n_{2}}$, thus the problem is transformed to a general symmetric bipartite matching problem and we can easily solve it by the method introduced in the previous subsection.

Secondly, if $n_{1}>n_{2}$, that is, the resource to be assigned is excessive for the users. In this case, if the users can not use the

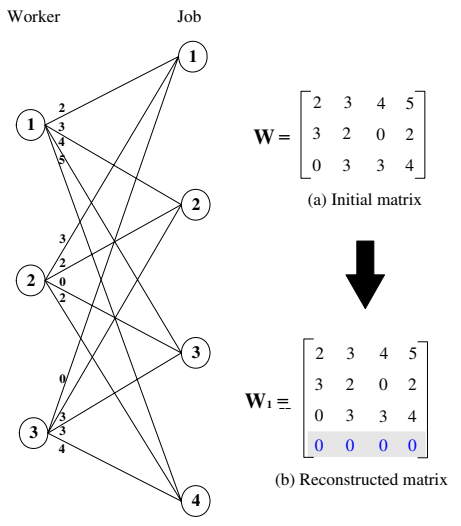

Fig. 2. Asymmetric bipartite matching (1).

redundant resource, as presented above, our MBM algorithm will append $\left(n_{1}-n_{2}\right)$ all-zeros columns to construct a square weight matrix and transform the problem to a symmetric bipartite matching one, as shown in Fig. 3. However, if the redundant resource may be used by the users, i.e. each user can accept multiple assignments simultaneously, then the previous method will cause resource wastage. In this case, our MBM is enhanced as follows:

1) Step 1: Duplicate and add the whole user set to $V_{2}$ till the new cardinality of $V_{2}$, denoted as $n_{2}^{\prime}$, is equal to or larger than the cardinality of $V_{1}$. Update the graph by adding the corresponding edges to the duplicated user sets. If $n_{2}^{\prime}=n_{1}$, go to step 3.

2) Step 2: If $n_{1}<n_{2}^{\prime},\left(n_{2}^{\prime}-n_{1}\right)$ all-zero rows are appended to form a square weight matrix.

3) Step 3: Solve the bipartite matching problem as a symmetric one by the method presented in the above subsection.

Fig. 4 illustrates the processing of MBM when all resource is usable for users. In the next section, an example will be given for both symmetric and asymmetric bipartite matchings.

\section{EXAMPLE}

In this section, MBM is applied for antenna assignments in the downlink of an MIMO communication system to maximize the overall channel capacity, while keeping the fairness among all mobile users.

\section{A. System Model}

The system model is shown in Fig. 5, where the base station has $N_{T}$ antennas and each mobile user has $N_{R}\left(\geq N_{T}\right)$ antennas. The number of mobile users is $K$. The transmit power is equally divided among the transmit antennas. The receiver estimates the post-detection signal-to-noise ratio (SNR) of each transmit antenna, and passes the SNR information to the base station through the uplink feedback channel at the start of each time-slot (of duration Ts). Based on this information, the scheduler selects a group of users and assigns the antennas to them to transmit in the time-slot. The channel encounters 


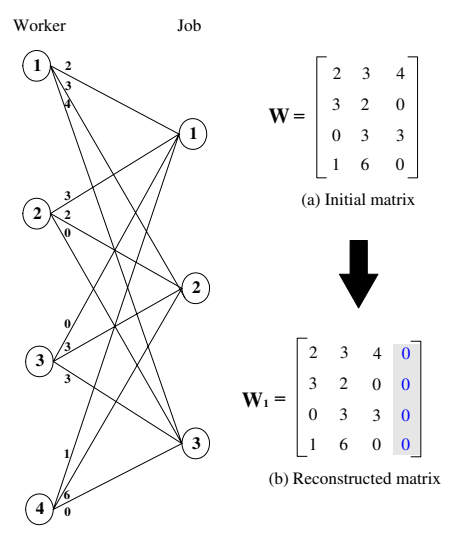

Fig. 3. Asymmetric bipartite matching (2).

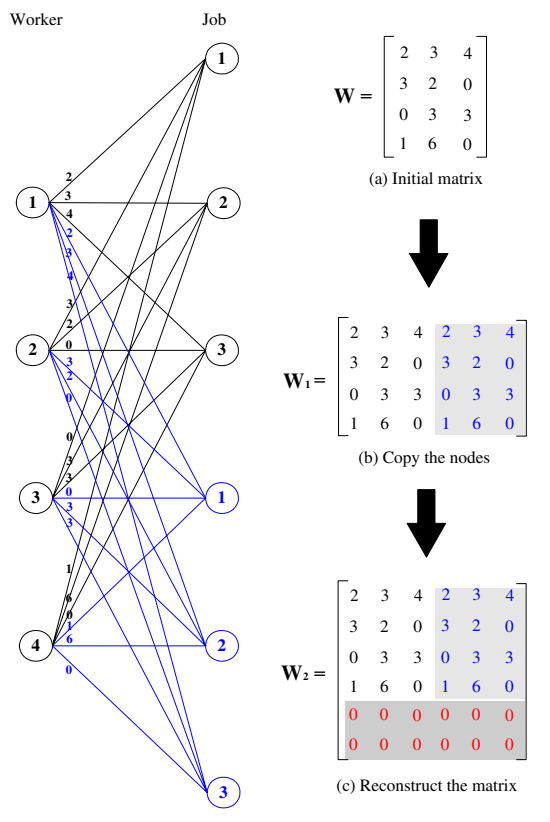

Fig. 4. Asymmetric bipartite matching (3).

path loss and shadow fading, and it is time-invariant during each time-slot and independent between time-slots. Also, the channel is assumed to be spatially uncorrelated. The channel matrix $H_{k}(t)$ between the base station and user $k$ at time-slot $t$ is [5]

$$
H_{k}(t)=\sqrt{S N R_{0} \cdot\left(l_{k} / L\right)^{-\beta} \cdot 10^{S_{k} / 10}} \cdot G_{k}(t),
$$

where $S N R_{0}$ is the median $S N R, L$ is the cell radius, $G_{k}(t)$ is an independent complex Gaussian random variable with zero mean and unit variance, $\beta$ is the path loss exponent, and $S_{k}(t)$ is a real Gaussian random variable with zero mean and

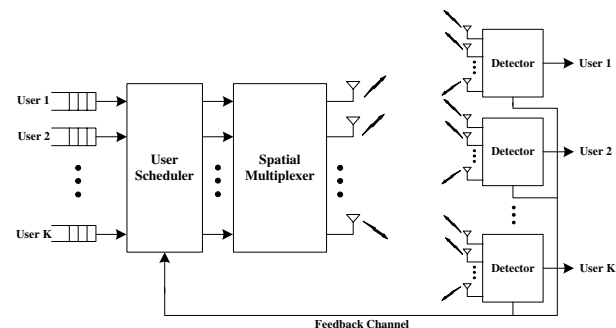

Fig. 5. Downlink MIMO system.

variance of $\sigma_{S}^{2}$. In addition, $l_{k}$ is the distance between the base station and user $k$. The distance of each user from the base station is randomly initialized between zero and $L$. The postdetection SNR [5] is defined as the SNR of a transmit symbol after minimum mean-squared error (MMSE) detection, and the corresponding value for the transmit antenna $n$ to user $k$ can be calculated as

$\gamma_{k, n}(t)=\frac{\left|\left[W_{k}(t) H_{k}(t)\right]_{n n}\right|^{2}}{\left(\sigma_{N}^{2} / P_{R}\right) N_{T} \sum_{m=1}^{N_{T}}\left|\left[W_{k}(t)\right]_{n m}\right|^{2}+\sum_{m=1, m \neq n}^{N_{T}}\left|\left[W_{k}(t) H_{k}(t)\right]_{n m}\right|^{2}}$,

where $P_{R}$ is the total received signal power and $\sigma_{N}^{2}$ is the noise power per receive antenna. The weight matrix for MMSE is given as

$$
W_{k}(t)=H_{k}^{H}(t)\left(H_{k}(t) H_{k}^{H}(t)+\left(\sigma_{N}^{2} N_{T} / P_{R}\right) I_{N_{R}}\right)^{-1},
$$

where $(\cdot)^{H}$ denotes the conjugate transpose and $I_{N_{R}}$ is the $N_{R} \times N_{R}$ identity matrix. Therefore, the system capacity for time slot $t$ can be expressed as

$$
C(t)=\sum_{n=1}^{N_{T}} \log _{2}\left(1+\gamma_{k, n}(t)\right)
$$

\section{B. Problem Formulation}

In spatial multiplexing, a data stream is split into multiple substreams, and each substream is transmitted through a transmit antenna. To exploit multiuser diversity, the scheduler can simultaneously choose as many multiple users as transmit antennas and allocate an antenna to each user. As the capacity is a function of SNR, maximizing the sum of the weight obtained from SNR is equivalent to the capacity maximization of (9). We further denote $C\left(k_{1}, \ldots, k_{N_{T}}\right)$ as the capacity achieved by allocating transmit antenna $i$ to user $k_{i}$ for $i=$ $1, \ldots, N_{T}$. Consequently, our proposed MBM aims to search $k_{1}, \ldots, k_{N_{T}}, \forall i, j(\neq i) ; k_{i} \neq k_{j}$ to

$$
\max C\left(k_{1}, \ldots, k_{N_{T}}\right)=\max \sum_{i=1}^{N_{T}} \log _{2}\left(1+\gamma_{k_{i}, i}\right)
$$


In a wireless communication system, the fairness index is widely used to evaluate the fairness performance [10]. It takes values between 0 and 1 , and is defined as follows:

$$
f=\frac{\left(\sum_{i=1}^{N_{T}} C_{k_{i}, i}\right)^{2}}{N_{T}\left(\sum_{i=1}^{N_{T}} C_{k_{i}, i}^{2}\right)},
$$

where $C_{k_{i}, i}=\log _{2}\left(1+\gamma_{k_{i}, i}\right)$ is the transmit rate achieved by assigning antenna $i$ to user $k_{i}$. We assume each user has the same QoS requirement on throughput. As presented in Section II-B, after each update of the graph $G$ by deleting the edge chosen by (4), a new maximum weight matching is obtained till there is no edge left in $G$. For each achieved matching $M_{j}$, the corresponding fairness index $f_{j}\left(j=1, \ldots, N_{T} \times\right.$ $K$ ) could be calculated by (11). Therefore the multiobjective optimization is achieved by choosing the maximum $f_{p}$ among the updated matchings, namely,

$$
\{p\}=\operatorname{argmax}_{j}\left\{f_{j}\right\}, j=1, \ldots, N_{T} \times K .
$$

Finally, the maximum matching $M_{p}$ should be the solution for the multiobjective capacity and fairness optimization problem.

In wireless MIMO systems, if $K=N_{T}$, a symmetric bipartite matching is easily formulated by MBM. However, in a real network, $K$ is not necessarily equal to $N_{T}$. If $K>N_{T}$, namely the channel resource is not adequate for all mobile users, some of users will not have channels in the current transmission slot. On the contrary, if $K<N_{T}$, that is the number of channels is larger than the number of mobile users, then multiple channels can be assigned to a user and the capacity of the user is the sum of the capacities on these multiple assigned channels. Therefore, such asymmetric bipartite matching can be solved by our proposed MBM and the bandwidth usage of the whole system is effectively increased.

\section{Simulation Results}

In this paper, we consider a $4 \times 4$ MIMO system, and evaluate the capacity and fairness performance for the best-effort traffics. We assume $S N R_{0}=10 \mathrm{~dB}$, the path loss exponent $\beta=3.7 \mathrm{~dB}$, the $\log$ standard deviation of shadow fading $\sigma_{S}=8 \mathrm{~dB}$, the cell radius $L=1 \mathrm{Km}$, and $P_{R} / \sigma_{N}^{2}=10 \mathrm{~dB}$. In this model, the distance of each user from the base station is uniformly distributed between 0 and $L$. Simulation time is $2 \times 10^{3}$ Ts. We compared the performance of the Hungarian maximum weighted matching, the pure fairness maximization scheme, and our proposed MBM algorithm. Table I compares the computational complexities of these three schemes. For MBM, given a certain fairness index requirement, $O\left(n^{6}\right)$ is the upper bound of the search complexity.

In the simulations, we assume $K=N_{T}$ for the symmetric bipartite matching. For the asymmetric bipartite matchings, we vary $K$ to 3 and 5 to test the performance of MBM. Fig. 6 shows the throughput performance comparisons for the symmetric bipartite matching case. For the generated network scenario, the Hungarian algorithm can provide the maximum
TABLE I

COMPUTATIONAL COMPLEXITIES

\begin{tabular}{|c|c|}
\hline Assignment scheme & Computational complexity \\
\hline \hline Hungarian Algorithm & $O\left(n^{4}\right)$ \\
\hline Pure fairness & $O(n !)$ \\
\hline MBM & $O\left(n^{6}\right)$ \\
\hline
\end{tabular}

system capacity of around $33.6 \mathrm{bps} / \mathrm{Hz}$ and a fairness index of around 0.77. If we assume the system fairness optimization target is to have a fairness index above 0.8, the Hungarian algorithm is inadequate. MBM can optimize the throughput for the given fairness requirements. We show the MBM throughput for different fairness index thresholds $(\xi)$ from 0.8 to 0.9 . By exhaustive search, the pure fairness maximization scheme gives the best fairness performance of over 0.9 , but it sacrifices the system capacity.

For the asymmetric bipartite matching, Fig. 7 shows the throughput performance comparisons when $K=5(K>$ $N_{T}$ ). The analysis is similar to the case above. The maximum throughput obtained by the Hungarian algorithm is around $44.8 \mathrm{bps} / \mathrm{Hz}$, but the fairness index is lower than 0.8 . Our MBM can improve the throughput, while satisfying different fairness requirements. Fig. 8 and Fig. 9 show the throughput performance when $K=3\left(K<N_{T}\right)$. In Fig. 8, we do not consider the utilization of the redundant antenna resource. The maximum throughput achieved by the Hungarian algorithm is around $30.3 \mathrm{bps} / \mathrm{Hz}$ and the fairness index is also lower than 0.8 . For the pure fairness maximization scheme, since the rate fluctuation of a mobile user is closely related to its location, the best performance of the fairness index is around 0.88. As shown in Fig. 8, for our MBM, if the fairness index threshold is 0.88 , the throughput performance is similar to the pure fairness maximization scheme. However our MBM can achieve higher fairness performance by abandoning certain edges at the cost of sacrificing the system capacity. In Fig. 9, we assume a user can receive signals from multiple antennas in the MIMO system, the throughput performance is greatly improved and much higher than the maximum throughput achieved by the Hungarian algorithm. In addition, as observed from Fig. 9, different fairness requirements can also be satisfied by our MBM algorithm.

\section{CONClusions}

In this paper, based on the Hungarian algorithm, a modified bipartite matching (MBM) algorithm is proposed for multiobjective optimization. This algorithm can effectively balance conflicting objectives in multiobjective optimization problems, and is widely applicable to resource assignment problems. In addition, MBM is enhanced to solve not only symmetric bipartite matching problems but also asymmetric problems. In this paper, we illustrate the application of MBM to antenna assignments in wireless MIMO systems. The simulation results show that MBM enjoys low computational complexity and maximizes the system capacity, while keeping the fairness 


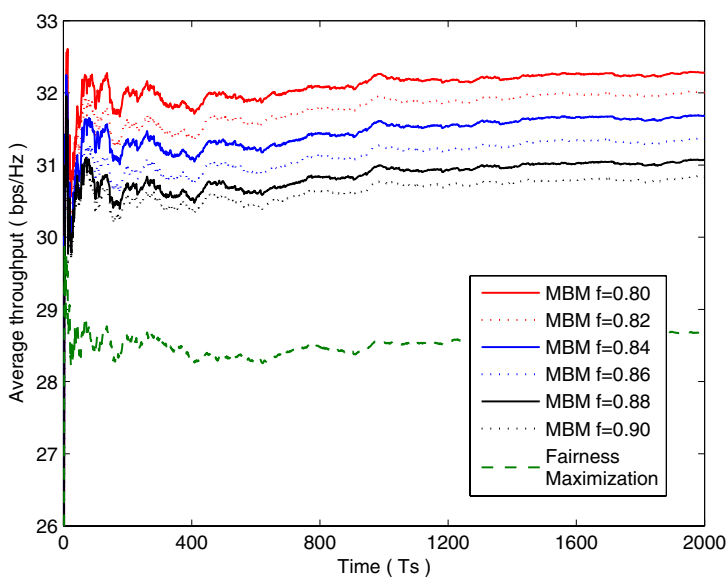

Fig. 6. Throughput comparisons for $K=N_{T}$.

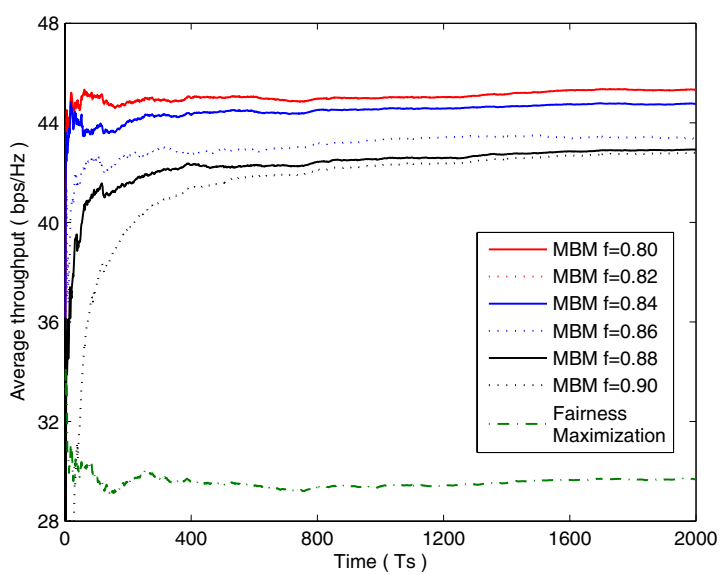

Fig. 7. Throughput comparisons for $K>N_{T}$.

among mobile users for both symmetric and asymmetric network scenarios.

\section{REFERENCES}

[1] H. W. Kuhn, "The Hungarian Method for the Assignment Problem," Naval Research Logistic Quarterly, vol. 2, pp. 83-97, 1955.

[2] K. J. Runser, J. M. Gorce, and S. Ubeda, "QoS Constrained Wireless LAN Optimization Within a Multiobjective Framework," IEEE Wireless Communcations, vol. 13, no. 6, pp. 26-33, 2006.

[3] B. Wu and Q. Wang, "A Multiobjective Analytic Framework for Slotted ALOHA Wireless LANs, " IEEE ICUPC, pp. 12-16, Tokyo, Japan, Nov. 1995.

[4] P. W. Wolniansky, C. J. Foschini, G. D. Golden, and R. A. Valenzuela, "V-BLAST: An architecture for Realizing High Data Rates over the Rich-scattering Wireless Channel," URSI Int Symp. Signals, Systems. ond Electronics, pp. 295-300, Pisa, Italy, Sept.-Oct. 1998.

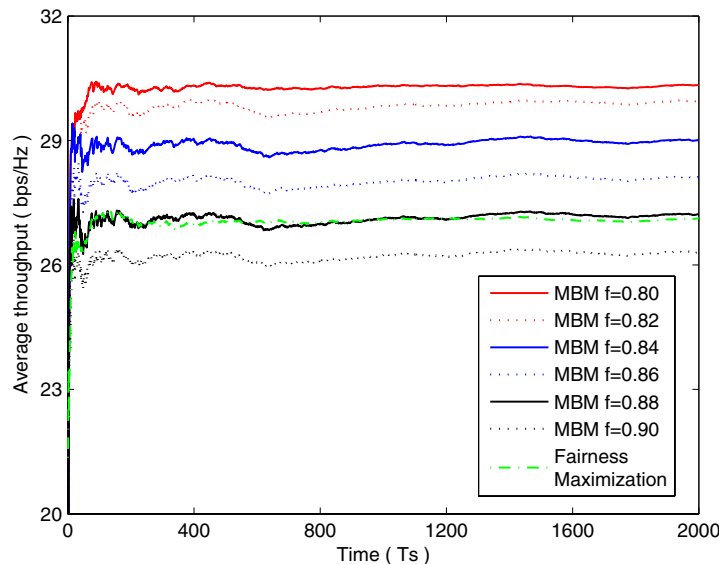

Fig. 8. Throughput comparisons for $K<N_{T}$ (1)

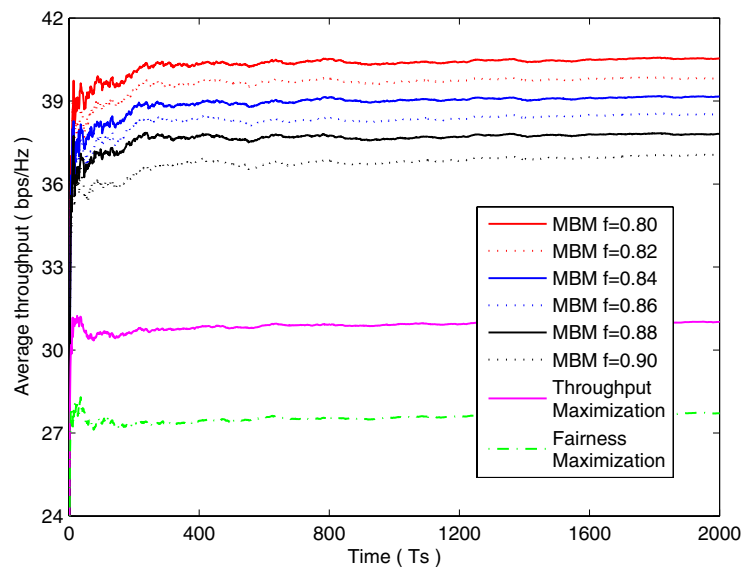

Fig. 9. Throughput comparisons for $K<N_{T}$ (2).

[5] R. W. Heath, M. Airy, and A. I. Paulraj, "Multiuser Diversity for MIMO Wireless Systems with Linear Receivers," Asilomor Conf. Signals, Systems, and Computers, pp. 1194-1199, Pacific Grove, CA, USA, Nov. 2001 .

[6] O. S. Shin and K. B. Lee, " Antenna-assisted Round Robin Scheduling for MIMO Cellular System," IEEE Communication Letters, vol. 7, no. 3, pp. 109-111, Mar. 2003.

[7] Y. J. Choi, J. Kim, and S. Bahk, "Downlink Scheduling with Fairness and Optimal Antenna Assignment for MIMO Cellular Systems," IEEE GLOBECOM, vol. 5, pp. 3165-3169, Dallas, Texas, USA, Nov. 2004.

[8] M. Torabzadeh and Y. Ji, "Efficient Assignment of Transmit Antennas for Wireless Communications," IEEE International Conference in Central Asia, pp. 1-5, Tashkent, Uzbekistan, Sept. 2006.

[9] D. B. West, Introduction to Graph Theory, Prentice Hall, 2001.

[10] R. Jain, D. Chiu, and W. Hawe, "A Quantitative Measure of Fairness and Discrimination for Resource Allocation in Shared Computer Systems," DEC Research, Tec. Report, TR-301, 1984. 